\title{
Upper limb dysfunction and activities in daily living in idiopathic normal pressure hydrocephalus
}

\author{
Jani Sirkka ${ }^{1}$ (1) Marita Parviainen ${ }^{1} \cdot$ Henna-Kaisa Jyrkkänen ${ }^{1} \cdot$ Anne M. Koivisto ${ }^{2,3,4} \cdot$ Laura Säisänen $^{5,6}$. \\ Tuomas Rauramaa $^{7} \cdot$ Ville Leinonen $^{1} \cdot$ Nils Danner $^{1}$
}

Received: 19 March 2021 / Accepted: 10 June 2021 / Published online: 8 July 2021

(c) The Author(s) 2021

\begin{abstract}
Background Idiopathic normal pressure hydrocephalus (iNPH) is a neurodegenerative disease with a characteristic symptom triad of gait disturbance, cognitive decline, and incontinence. Recently, also dysfunctions in upper limbs have been described in iNPH and reported to improve after shunt surgery. We aim to describe the role of upper limb motor function in the clinical assessment of iNPH patients and its influence on activities of daily living (ADL).

Methods Seventy-five consecutive patients with probable iNPH were studied pre-operatively and at 3 and 12 months after shunt surgery. The pre-operative evaluation included lumbar drainage of cerebrospinal fluid (tap test). Motor functions were assessed in upper and lower limbs with Grooved Pegboard Test (GPT), Box \& Block Test (BBT), Total Score of Gait (TSG), and balance test. ADL was assessed with Barthel's index and cognition in accordance with the Consortium to Establish a Registry for Alzheimer's Disease (CERAD).

Results Patients showed improvement in all motor tests and ADL at 3 months after shunt surgery. The improvement remained stable during the 12-month post-operative follow-up. The motor function tests correlated with each other and with ADL.

Conclusions A 3-month follow-up period after shunt surgery is adequate to show improvement in motor tasks, and a positive outcome will last for at least 12 months. A shunt-responsive dysfunction of upper limb motor performance plays a major role in ADL of iNPH patients. Therefore, we suggest an evaluation of upper limb motor performance to be included in routine evaluation of iNPH patients.
\end{abstract}

Keywords Idiopathic normal pressure hydrocephalus $\cdot$ Symptoms $\cdot$ Upper limb motor function $\cdot$ Shunt surgery $\cdot$ Activities of daily living

\section{Introduction}

Idiopathic normal pressure hydrocephalus (iNPH) is a neurodegenerative disease with a clinical symptom triad of disturbed gait, declined cognition, and incontinence [1]. The

This article is part of the Topical Collection on CSF Circulation

Jani Sirkka

janisir@uef.fi

1 Department of Neurosurgery, Kuopio University Hospital and Institute of Clinical Medicine - Neurosurgery,

University of Eastern Finland, P.O. Box 100,

70029 KYS Kuopio, Finland

2 Department of Neurology, Neuro Center, Kuopio University Hospital, Kuopio, Finland

3 Department of Neurology, Institute of Clinical Medicine, School of Medicine, Faculty of Health Sciences, University of Eastern Finland, Kuopio, Finland only effective treatment, shunt surgery, is based on diversion of cerebrospinal fluid (CSF) [2,3].

Disturbed gait is usually the most prominent symptom of iNPH and plays also a major diagnostic role $[2,4]$. The role of upper limbs has received considerably less attention despite some early evidence on their involvement in

4 Department of Geriatrics/ Internal Medicine and Rehabilitation, Helsinki University Hospital and Department of Neurosciences, Faculty of Medicine, University of Helsinki, Helsinki, Finland

5 Clinical Neurophysiology, Imaging Center, Kuopio University Hospital, Kuopio, Finland

6 Department of Applied Physics, University of Eastern Finland, Kuopio, Finland

7 Department of Pathology, Kuopio University Hospital and Institute of Clinical Medicine - Pathology, University of Eastern Finland, Kuopio, Finland 
the clinical characteristics of iNPH [5]. In parallel with gait, upper limb motor function has been proven to improve with shunt surgery or after CSF drainage [6-10]. Subsequently, clinical evaluation of iNPH patients has been suggested to be supplemented with testing of upper limbs [11]. However, the effect of upper limb dysfunction on daily living of iNPH patients is unknown and patients may also experience subjective benefits from shunt surgery even if there is no objective improvement on the iNPH grading scale [12].

Therefore, we aim to characterize associations between upper limb motor function, gait, balance, cognition, and activities of daily living (ADL) and to evaluate their responsiveness to shunt surgery.

\section{Methods}

\section{Study population}

The study population was recruited prospectively from the neurosurgical outpatient clinic of Kuopio University Hospital (KUH) from May 2017 to December 2019. All patients had previously undergone a neurological evaluation and were referred to KUH for neurosurgical evaluation due to possible iNPH. Patients had one to three symptoms related to iNPH (impaired gait, declined cognition, or urinary incontinence) together with brain imaging finding of enlarged brain ventricles. During the study period, the total number of patients scheduled for operative treatment was 84 ( 39 women and 45 men, mean age 75.0 years \pm 5.8 years, range $61-86$ years). The flow chart of the study is presented in Fig. 1.

\section{Motor function assessments}

Motor function was assessed in accordance with an assessment scale for clinical evaluation of iNPH [13] in three different domains: gait, balance, and upper limb performance. Gait was evaluated by a $10-\mathrm{m}$ walking test, which was repeated three times. The used time, number of steps, and pace were scored from 0 to 100 , and then the total score of the gait (TSG) was calculated as an average of these sections. Balance was tested by asking the subject to stand still for $30 \mathrm{~s}$ in different positions and scored from 0 to 100 . Upper limb fine motor performance was evaluated with the Grooved Pegboard Test (GPT) in which the subject places key shaped pegs in holes with randomly positioned slots. GPT was scored from 0 to 100 depending on the used time. In addition, upper limb gross motor function was tested with the Box \& Block Test (BBT). In BBT, the subject moves as many cubes $(2.5 \times 2.5 \times 2.5 \mathrm{~cm})$ as possible from one box to another within a $60 \mathrm{~s} \mathrm{time} \mathrm{limit} \mathrm{[14].}$

\section{Activities of daily living and cognition}

Activities of daily living (ADL) were assessed with the Barthel's index (BI) with the following domains (the maximum score of each section is indicated in the brackets): feeding (10), transfers (15), grooming (5), toilet use (10), bathing (5), mobility (15), stair walk (10), dressing (10), bowel control (10), and bladder control (10) giving a maximum score of 100 [15]. An experienced healthcare professional scored the BI by interviewing and observing the ability to function in the above-mentioned sections. The level of cognition was assessed in accordance with the Consortium to Establish a Registry for Alzheimer's Disease CERAD [16]. The Finnish version of CERAD includes nine subtests: Verbal fluency, 15-Items Boston Naming test, Mini-Mental State Exam (MMSE), Word list learning, Word list recall, Word list recognition, Constructional praxis, Delayed constructional praxis, and Clock drawing [17].

\section{Tap test}

As a part of routine pre-operative evaluation, every patient underwent a tap test in which CSF was drained up to $40 \mathrm{ml}$ by lumbar puncture (LP). The purpose of the tap test was to detect possible immediate improvement in gait which may predict a positive outcome of shunt surgery $[18,19]$. The gait test was repeated identically 30-60 min after the LP.

\section{Surgical treatment}

The decision of surgical treatment was made by an experienced neurosurgeon of KUH. The decision was based on the symptom triad, imaging findings and tap test outcome in accordance with the KUH iNPH protocol [12]. In all operated patients, ventriculoperitoneal shunting was performed with an adjustable valve.

\section{Brain biopsy and immunohistochemistry}

During shunt surgery, 1-3 cortical brain biopsies of 2-5 mm in diameter and 3-7 $\mathrm{mm}$ in length were obtained using a biopsy needle. The biopsies were taken prior to and using the same route as for placing the ventricular catheter (anterior to the coronal suture and $3 \mathrm{~cm}$ from midline). The detailed procedure of the immunohistochemical analysis has been described previously [20]. From all samples, a neuropathologist analyzed the presence of the immunoreactivity for hyperphosphorylated tau $(\mathrm{HP} \tau)$ and amyloid-beta $(\mathrm{A} \beta)$ using light microscopy. Patients were then further categorized into two subgroup by the presence of pathology of the $\mathrm{HP} \tau$ and/ or $\mathrm{A} \beta$ and the absence of pathology of the HP $\tau$ and $\mathrm{A} \beta$. 
Fig. 1 Flow chart of the study ETV Endoscopic third ventriculostomy, FTD frontotemporal dementia, PD Parkinson's disease, CERAD Consortium to Establish a Registry for Alzheimer's Disease

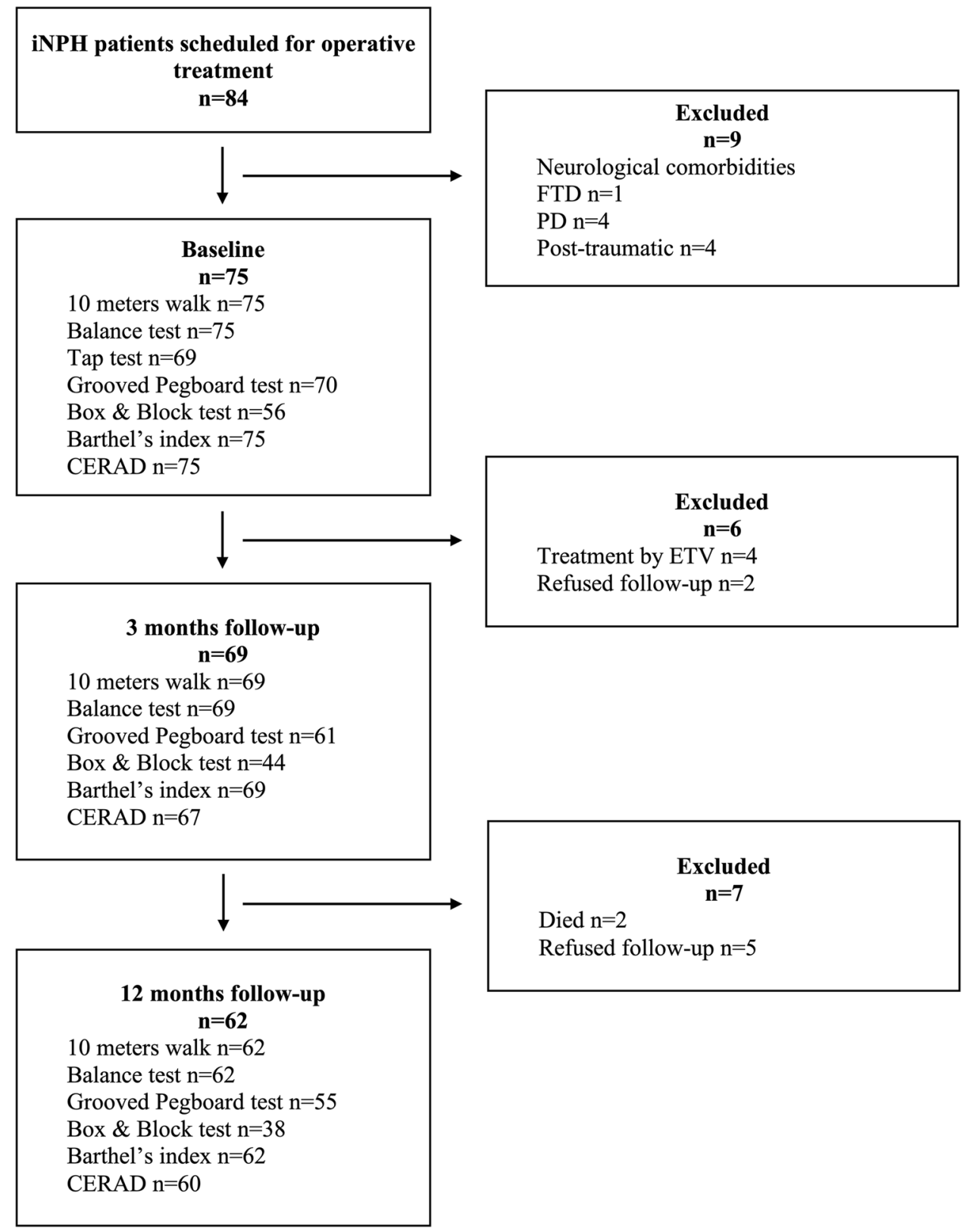

\section{Follow-up}

All clinical tests were performed at the outpatient clinic. The baseline tests were performed prior to the TAP test and repeated at 3 and 12 months after shunt surgery (Fig. 1).

\section{Statistical analysis}

Statistical analyses were performed with SPSS (version 24.4; IBM Corporation, Somers, NY). The normality of distribution in each variable was ensured with the Kolmogorov-Smirnov and Shapiro-Wilk tests; CERAD and BBT were normally distributed and other parameters were non-normally distributed. Patients with missing values were excluded from longitudinal and correlation analyses for that parameter (Fig. 1). Baseline and follow-up scores of CERAD and BBT were compared using repeated measures T-test, and parameters with highly skewed distribution (TSG, balance, GPT and BI) were analyzed using Wilcoxon signed-rank test. TSG scores before and after the TAP test were also compared using Wilcoxon signed-rank test. In each test a positive change of one or more points at followup was defined as improvement. Linear regression was used to determine differences in shunt surgery outcomes between subgroups. The surgical outcome was defined as a difference between baseline and follow-up scores of each parameter. Age and sex were standardized in linear regression model. Spearman's test was used for correlation analyses at baseline 
and follow-ups except for CERAD and BBT for which Pearson's test was used. $p$ values $<0.05$ were considered as statistically significant.

\section{Results}

There was significant correlation of upper and lower limb motor function with each other and with cognition (Table 1). At baseline, TSG correlated significantly with GPT and BBT. GPT and BBT exhibited also a significant correlation with each other. BI correlated significantly with all motor function tests and CERAD. CERAD correlated with all motor function tests except balance. At 3-month follow-up after shunt surgery, all parameters correlated with each other. At 12-month follow-up motor function tests except balance and BBT correlated with each other and with CERAD. BI correlated with TSG, balance and CERAD.

In the tap test TSG improved in 52/65 (80.0\%) patients $(p<0.001)$ (Fig. 1). The improvement correlated with the final TSG at follow-up ( $\mathrm{r}=0.367, p=0.003)$ but not with changes in GPT or in BBT. Three months after shunt surgery, the TSG score was higher than after the tap test

Table 1 Correlations at baseline, 3-month follow-up, and 12-month follow-up

\begin{tabular}{llllll}
\hline Baseline & & & & & \\
\hline & Balance & GPT & BBT & BI & CERAD \\
\hline TSG & $0.613^{* *}$ & $0.470^{* *}$ & $0.435^{* *}$ & $0.705^{* *}$ & $0.322^{* *}$ \\
Balance & & $0.522^{* *}$ & $0.413^{*}$ & $0.586^{* *}$ & 0.217 \\
GPT & & & $0.615^{* *}$ & $0.482^{* *}$ & $0.445^{* *}$ \\
BBT & & & & $0.496^{* *}$ & $0.306^{*}$ \\
BI & & & & & $0.298^{*}$
\end{tabular}

\begin{tabular}{|c|c|c|c|c|c|}
\hline \multicolumn{6}{|c|}{ 3-month follow-up } \\
\hline & Balance & GPT & BBT & BI & CERAD \\
\hline TSG & $0.657 * *$ & $0.615^{* *}$ & $0.471 * *$ & $0.566 * *$ & $0.495^{* *}$ \\
\hline Balance & & $0.657 * *$ & $0.500 * *$ & $0.530 * *$ & $0.407 * *$ \\
\hline GPT & & & $0.721 * *$ & $0.570 * *$ & $0.604 * *$ \\
\hline BBT & & & & $0.670 * *$ & $0.677 * *$ \\
\hline BI & & & & & $0.503 * *$ \\
\hline \multicolumn{6}{|c|}{ 12-month follow-up } \\
\hline & Balance & GPT & BBT & BI & CERAD \\
\hline TSG & $0.568 * *$ & $0.409 * *$ & $0.482 *$ & $0.563 * *$ & $0.569 * *$ \\
\hline Balance & & $0.404 * *$ & 0.223 & $0.334 * *$ & $0.358 * *$ \\
\hline GPT & & & $0.459 * *$ & 0.197 & $0.633 * *$ \\
\hline BBT & & & & 0.240 & $0.480 * *$ \\
\hline BI & & & & & $0.332 * *$ \\
\hline
\end{tabular}

${ }^{*} p<0.05$ and $* * p<0.01$

BBT $=$ Box $\&$ Block test, GPT $=$ Grooved Pegboard test, $\mathrm{TSG}=$ Total score of gait, $\mathrm{BI}=$ Barthel's index $(p=0.003)$, and at 12-month follow-up it increased even further $(p=0.005)$. In addition, balance score $(p<0.001)$, GPT $(p<0.001)$, BBT $(p=0.002)$, BI $(p<0.001)$, and CERAD $(p=0.002)$ were significantly higher at 3 -month follow-up after shunt surgery than at baseline (Fig. 2 and Table 2). Improvement rates of test scores at 3-month follow up are shown in Table 2. Changes in each BI domain are illustrated in Fig. 3. Cortical brain biopsy was obtained from 63 patients. Thirty-three patients exhibited normal findings in the immunohistochemical analysis, whereas 30 patients had $\mathrm{A} \beta$ and 7 patients had $\mathrm{HP} \tau$. There were no significant differences in outcomes of shunt surgery between patients who had cortical brain pathology (A $\beta$ and/or HP $\tau$ ) and those who had not (Table 2).

At baseline, three patients were not able to complete the walking test and two patients were not able to perform GPT. However, even at baseline upper limb function could be evaluated in all patients using BBT. After shunt surgery, all but one patient managed to complete the walking test, and all patients were able to perform GPT.

\section{Discussion}

This prospective cohort study aimed to extensively characterize the motor performance of iNPH patients and the response to shunt surgery. We found that the motor impairment in iNPH is not limited to the classically reported gait problems but extends to comprehensive motor impairment also in the upper limbs. In tandem with gait, the impairment in upper limb motor function seems to be reversible in nature and to respond well to shunt surgery. Furthermore, the motor performance of iNPH patients seems to be closely related to ADL functions.

\section{Follow-up time}

Current iNPH guidelines recommend assessing short-term outcomes of shunt surgery for up to 12 months [21, 22]. In our study population, an improvement in all the applied motor function tests was observed at the first follow-up 3 months after shunt surgery. Of all studied parameters, only CERAD and TSG showed slight further improvement between the 3-and 12-month follow-ups. This indicates that a 3-month follow-up is sufficient to show the potential of improvement after shunt surgery. Most importantly, no worsening of the motor symptoms or cognitive decline was observed during 1-year follow-up.

\section{Brain pathology}

Interestingly, the presence of $\mathrm{HP} \tau$ and/or $\mathrm{A} \beta$ in cortical brain biopsy had no impact on the surgical outcome with 
Fig. 2 Scores of individual tests at baseline and at follow-ups means and standard deviations of test scores at baseline and at 3- and 12- month follow-ups after shunt surgery

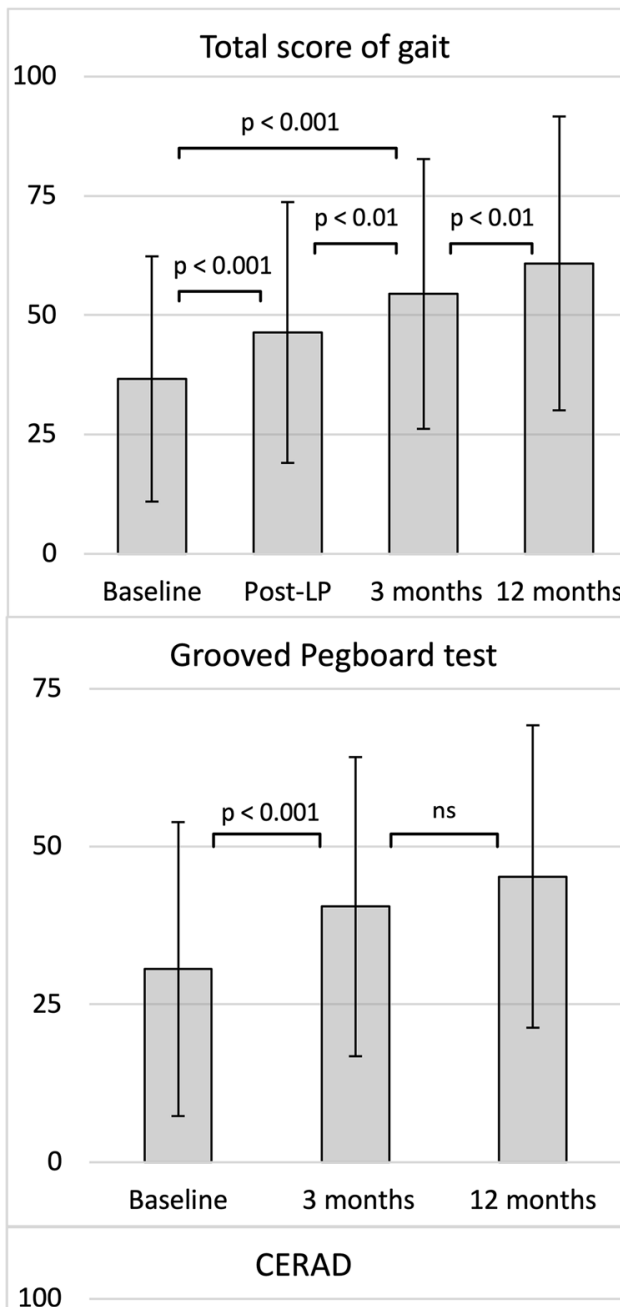

100

Balance score

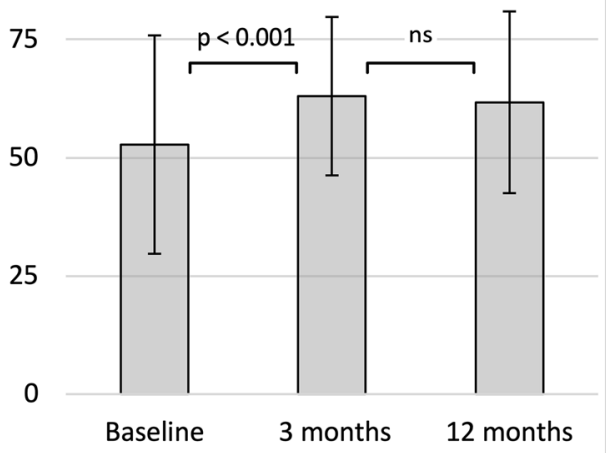

75

Box \& Block test

5

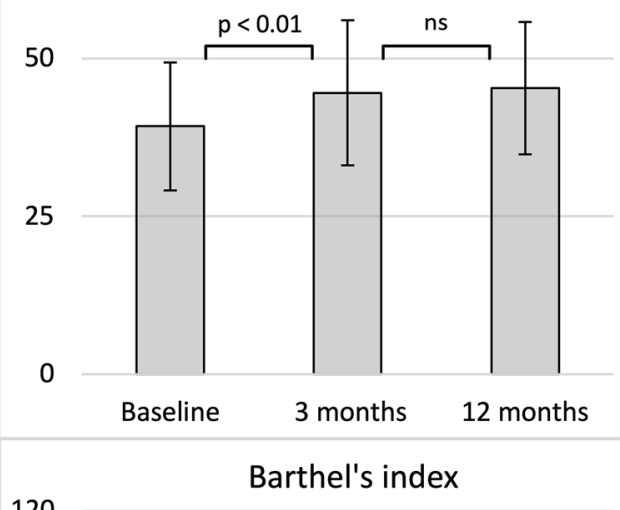

120

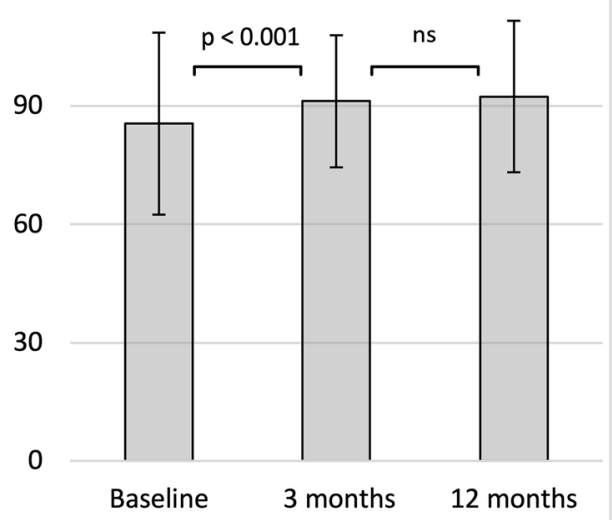

regard to motor function, cognition, or ADL. Alzheimer disease-related pathology in brain biopsy (HP $\tau$ and $A \beta$ ) seems to be frequent in iNPH and should not been used to exclude patients from shunt surgery [23]. This group of patients should be followed up in clinical studies in order to determine, whether the prognosis differs from classical iNPH.

\section{Upper limb testing}

In previous studies, an improvement of upper limb motor function in GPT has been reported at 3 months after shunt surgery and found to remain stable during a 12-month follow-up [11, 24]. Our current results support these findings. However, we found a significant improvement in BBT, a 
Table 2 Summary of shunt surgery outcomes
Fig. 3 Domains of Barthel's index in patients with improved total score the grey dotted line represents the mean of baseline values. The black solid line represents the mean of three-month follow-up values. The grey area represents the scale of Barthel's index, ${ }^{*} \mathrm{p}<0.05$ and $* * \mathrm{p}<0.01$ (difference between baseline and three-month follow-up)

\begin{tabular}{lllll}
\hline & Baseline & 3 months & 12 months & Improvement rate $^{\mathbf{a}}$ \\
\hline TSG & $36.7 \pm 25.7$ & $54.5 \pm 28.3$ & $60.9 \pm 30.8$ & $85.6 \%(59 / 69)$ \\
Biopsy+ & $32.2 \pm 24.1$ & $46.9 \pm 23.7$ & $53.9 \pm 26.8$ & $82.8 \%(24 / 29)$ \\
Biopsy- & $41.7 \pm 28.4$ & $60.3 \pm 30.7$ & $67.4 \pm 32.3$ & $84.4 \%(27 / 32)$ \\
Balance & $52.8 \pm 23.1$ & $63.0 \pm 16.8$ & $61.7 \pm 19.2$ & $39.1 \%(27 / 69)$ \\
Biopsy+ & $46.8 \pm 26.1$ & $60.6 \pm 22.0$ & $57.6 \pm 22.8$ & $48.3 \%(14 / 29)$ \\
Biopsy- & $54.8 \pm 21.4$ & $64.8 \pm 12.8$ & $63.3 \pm 16.3$ & $40.6 \%(13 / 32)$ \\
GPT & $30.6 \pm 23.3$ & $40.5 \pm 23.7$ & $45.3 \pm 23.9$ & $57.4 \%(35 / 61)$ \\
Biopsy+ & $30.7 \pm 22.7$ & $38.9 \pm 21.9$ & $42.0 \pm 21.6$ & $48.1 \%(13 / 27)$ \\
Biopsy- & $29.7 \pm 23.7$ & $42.2 \pm 24.4$ & $47.9 \pm 24.5$ & $66.7 \%(18 / 27)$ \\
BBT & $39.3 \pm 10.1$ & $44.6 \pm 11.5$ & $45.3 \pm 10.5$ & $72.7 \%(32 / 44)$ \\
Biopsy+ & $40.2 \pm 9.7$ & $45.0 \pm 10.1$ & $45.7 \pm 10.2$ & $70.8 \%(17 / 24)$ \\
Biopsy- & $37.2 \pm 11.3$ & $44.4 \pm 14.1$ & $45.2 \pm 12.0$ & $81.3 \%(13 / 16)$ \\
BI & $85.6 \pm 17.3$ & $91.2 \pm 13.4$ & $92.4 \pm 11.2$ & $49.3 \%(34 / 69)$ \\
Biopsy+ & $80.3 \pm 22.3$ & $90.2 \pm 14.5$ & $91.7 \pm 8.7$ & $55.2 \%(16 / 29)$ \\
Biopsy- & $88.8 \pm 11.9$ & $91.6 \pm 14.2$ & $93.3 \pm 13.2$ & $40.6 \%(13 / 32)$ \\
CERAD & $60.1 \pm 11.9$ & $63.5 \pm 12.5$ & $65.7 \pm 13.1$ & $64.2 \%(43 / 67)$ \\
Biopsy+ & $58.5 \pm 11.5$ & $62.1 \pm 11.4$ & $63.0 \pm 13.4$ & $64.3 \%(18 / 28)$ \\
Biopsy- & $61.6 \pm 13.1$ & $64.4 \pm 14.2$ & $68.6 \pm 13.1$ & $59.4 \%(19 / 31)$ \\
\hline
\end{tabular}

${ }^{\mathrm{a}}$ Improvement of one or more points in the test score at 3-month follow-up

Biopsy $+=$ subgroup with hyperphosphorylated tau and/or amyloid-beta (A $\beta)$ in cortical brain biopsy

Biopsy-= subgroup without hyperphosphorylated tau or amyloid-beta $(\mathrm{A} \beta)$ in cortical brain biopsy

BBT $=$ Box \& Block test, GPT $=$ Grooved Pegboard test, TSG = Total score of gait, BI = Barthel's index

Means and standard deviations of test scores at baseline and at 3- and 12- month follow-ups after shunt surgery

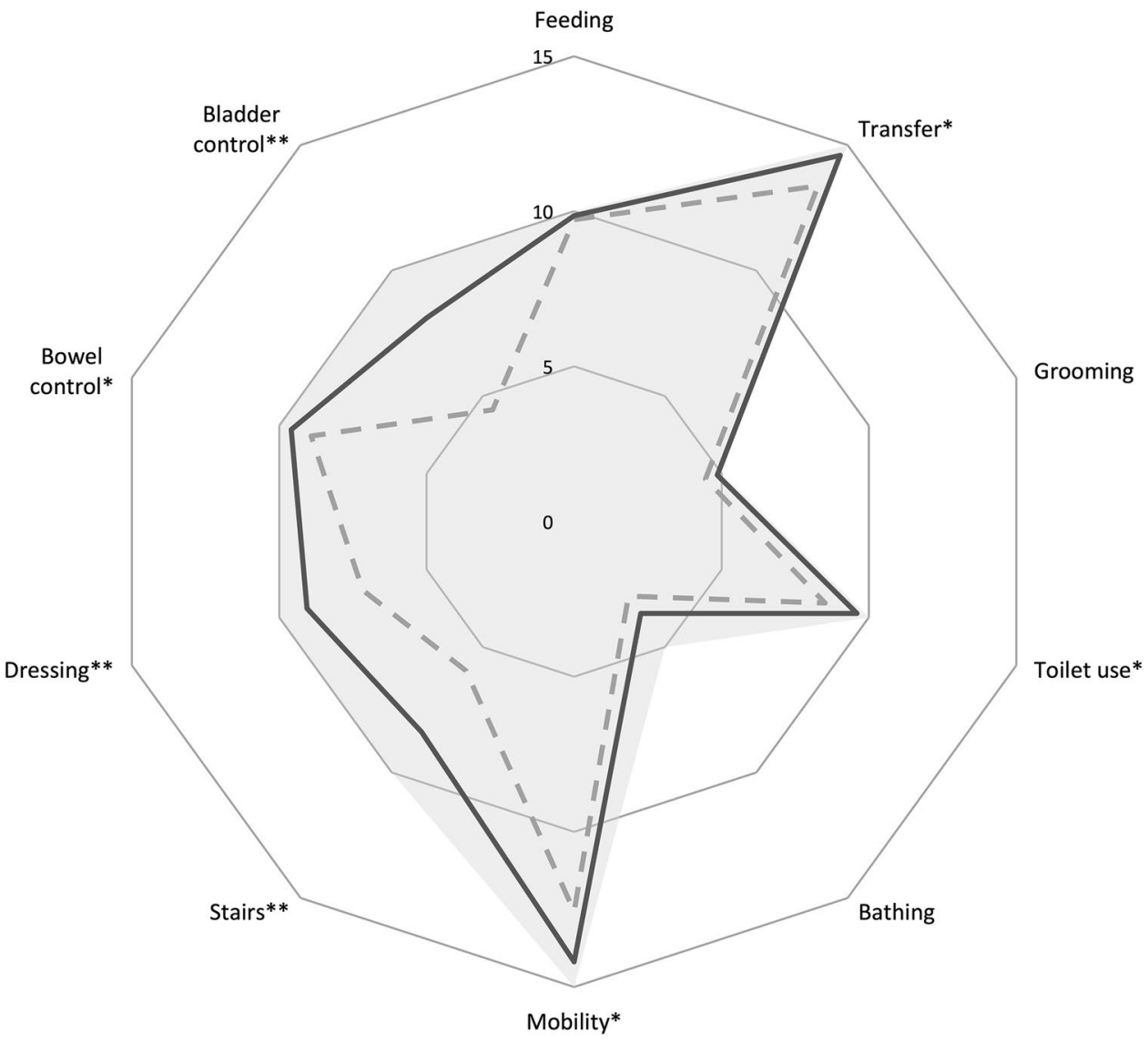


novel test in iNPH, in an even higher proportion of patients than in the previously reported GPT. BBT is used to assess simple gross-motor manual dexterity, and compared with GPT, it does not require as complex and accurate psychomotor and visual performance [25]. Therefore, it may be a more suitable test for patients with severe symptoms. The present study population included three patients with extremely severe motor symptoms who could not walk at all and two patients who could not perform GPT at baseline. Nevertheless, every patient was able to complete the BBT. In addition, unlike in BBT, there is a floor effect in the $10-\mathrm{m}$ walking tests and GPT, commonly used in iNPH, which complicates their usage in extremely poor performing subjects [7].

\section{Preoperative evaluation}

In the preoperative assessment, up to $80 \%$ of patients exhibited a positive tap test result (improvement) in TSG, and the improvement rate was even higher at 3 months after shunt surgery. Thus, the tap test result in TSG seems to be highly indicative of the improvement in TSG after shunt surgery. Some previous studies suggest that CSF drainage via LP might also improve upper limb motor function [6,7]. The present results show a significant improvement in upper limb motor function after shunt surgery. However, the improvement of TSG in the tap test is not associated with improvement in GPT or in BBT after shunt surgery. This indicates that there could be improvement in upper limb motor function even if gait does not improve in the tap test. On the other hand, it has been shown that manual dexterity might not improve acutely after LP [26, 27]. Therefore, the predictive role of upper limb motor function tests as a part of the preoperative TAP test is controversial and not generally used in clinical practice $[28,29]$. The underlying dysfunction and the effect of CSF drainage may differ between upper and lower limbs as suggested in our recent study, in which an initial change in corticospinal excitability after decreased intrathecal pressure was associated with improved walking speed but not with upper limb function [30].

\section{Activities of daily living}

In the present study, ADL functions were good in most patients at baseline but still showed improvement at the 3-month follow-up after shunt surgery, and the good outcome lasted for at least 12 months. Expectedly, improvement in the BI was mainly observed in domains related to gait, such as mobility or stair walking and domains of continence. Interestingly, dressing seemed to be among the domains with the highest improvement, which is likely to reflect the motor function of the upper limbs. The BI was also highly associated with all motor function tests both at baseline and at follow-up, which emphasizes the role of motor symptoms in the ADL functions of iNPH patients. The improvement in ADL with shunt surgery is an invaluable result, since patients with iNPH have been reported to function worst as compared with other types of adult hydrocephalus [31]. Even a small improvement of ADL functions might improve the quality of life in patients with iNPH [32], which has been reported even in the absence of objective benefit of shunt surgery in other clinical tests [33]. One previous study has also demonstrated that the improvement in ADL functions could last up to 5 years in iNPH patients with a favorable clinical or subjective outcome of surgical treatment [34]. Hence, improving and maintaining ADL functions with proper treatment is meaningful for patients, their relatives, and even from a socioeconomic point-of-view [35].

\section{Conclusions}

This is the first study to evaluate upper limb motor function with BBT and to characterize the relationship between various motor parameters and ADL functions. A positive shunt response was observed in gait, balance, upper limb motor functions, ADL and cognition. ADL functions correlated strongly with both upper and lower limb motor function. Patients with comorbid non-iNPH-related walking difficulties may especially benefit from using upper limb function in the preoperative evaluation for shunt surgery. It remains to be shown, whether a sub-population of iNPH patients might benefit from shunt surgery in terms of upper limb motor performance even if the preoperative workup does not imply a positive effect on gait. We suggest that upper limb motor function testing should be included in routine pre-operative evaluation and post-operative follow-up of iNPH patients.

\section{Limitations and generalizability}

Despite the study setting of a prospective cohort with very limited exclusion criteria, the patients were able to perform surprisingly well in ADL based on the BI scores. Thus, the results cannot be unquestionably generalized to severely affected individuals. Of the applied parameters only TSG and GPT have been validated as a part of the clinical assessment scale of iNPH [13]. Upper limb motor function was assessed at baseline and at postoperative follow-ups but the role of BBT and GPT as part of preoperative TAP test remains to be elucidated. Due to the clinical setting of the study, all tests could not be carried out in all patients at every time point. The follow-up period of the current study was limited to 1 year. Future studies are warranted to show the longevity of the shunt response in upper limb motor performance. 
Abbreviations iNPH: Idiopathic normal pressure hydrocephalus; CSF: Cerebrospinal fluid; ADL: Activities of daily living; ETV: Endoscopic third ventriculostomy; FTD: Frontotemporal dementia; PD: Parkinson's disease; KUH: Kuopio University Hospital; TSG: Total score of the gait; GPT: Grooved Pegboard test; BBT: Box \& Block Test; BI: Barthel's index; LP: Lumbar puncture; $\mathrm{HP} \tau$ : Hyperphosphorylated tau; A $\beta$ : Amyloid-beta; ns: Non-significant difference

Authors' contributions Jani Sirkka, MB, Kuopio University Hospital and University of Eastern Finland, Designed and conceptualized study, interpreted and analyzed data, wrote first draft.

Marita Parviainen, RN, Kuopio University Hospital and University of Eastern Finland, Acquired data, revised manuscript.

Henna-Kaisa Jyrkkänen MD, PhD, Kuopio University Hospital and University of Eastern Finland, Acquired data, revised manuscript.

Anne M Koivisto MD, PhD, Kuopio University Hospital and University of Eastern Finland, Designed and conceptualized study, revised manuscript.

Laura Säisänen, $\mathrm{PhD}$, Kuopio University Hospital and University of Eastern Finland, Revised manuscript, supervised study.

Tuomas Rauramaa, MD, PhD, Kuopio University Hospital and University of Eastern Finland, Acquired data, revised manuscript.

Ville Leinonen, MD, PhD, Kuopio University Hospital and University of Eastern Finland, Designed and conceptualized study, acquired data, revised manuscript, supervised study.

Nils Danner, MD, PhD, Kuopio University Hospital and University of Eastern Finland, Designed and conceptualized study, acquired and interpreted data, revised manuscript, supervised study.

Funding Open access funding provided by University of Eastern Finland (UEF) including Kuopio University Hospital. JS has received funding from The Finnish Brain Foundation (Helsinki, Finland), The Maire Taponen Foundation (Espoo, Finland), The Cultural Foundation of Northern Savo (Kuopio, Finland), The Uulo Arhio Memorial Fund (Helsinki, Finland), The Päivikki and Sakari Sohlberg Foundation (Helsinki, Finland) and The Finnish Medical Foundation (Helsinki, Finland). LS was supported by Academy of Finland (grant number 322423).

\section{Declarations}

Ethics approval The study was approved by the Research Ethics Committee of the Northern Savo Hospital District, 276/2016.

Consent to participate All patients provided written informed consent.

Consent for publication All authors have approved the manuscript for publication.

Conflict of interest ND has attended a course sponsored by B Braun, distributor of Miethke shunt systems. Other authors report no disclosures relevant to the manuscript.

Open Access This article is licensed under a Creative Commons Attribution 4.0 International License, which permits use, sharing, adaptation, distribution and reproduction in any medium or format, as long as you give appropriate credit to the original author(s) and the source, provide a link to the Creative Commons licence, and indicate if changes were made. The images or other third party material in this article are included in the article's Creative Commons licence, unless indicated otherwise in a credit line to the material. If material is not included in the article's Creative Commons licence and your intended use is not permitted by statutory regulation or exceeds the permitted use, you will need to obtain permission directly from the copyright holder. To view a copy of this licence, visit http://creativecommons.org/licenses/by/4.0/.

\section{References}

1. Relkin N, Marmarou A, Klinge P, Bergsneider M, Black PM (2005) Diagnosing idiopathic normal-pressure hydrocephalus. Neurosurgery 57(3 Suppl):S4-16

2. McGirt MJ, Woodworth G, Coon AL, Thomas G, Williams MA, Rigamonti D (2005) Diagnosis, treatment, and analysis of longterm outcomes in idiopathic normal-pressure hydrocephalus. Neurosurgery 57(4):699-705

3. Kazui H, Miyajima M, Mori E, Ishikawa M (2015) SINPHONI-2 Investigators. Lumboperitoneal shunt surgery for idiopathic normal pressure hydrocephalus (SINPHONI-2): an open-label randomised trial. Lancet Neurol 14(6):585-94. https://doi.org/10. 1016/S1474-4422(15)00046-0

4. Marmarou A, Young HF, Aygok GA, Sawauchi S, Tsuji O, Yamamoto $T$ et al (2005) Diagnosis and management of idiopathic normal-pressure hydrocephalus: a prospective study in 151 patients. J Neurosurg 102:987-997. https://doi.org/10.3171/jns.2005.102.6. 0987

5. SoelbergSørensen P, Jansen EC, Gjerris F (1986) Motor disturbances in normal-pressure hydrocephalus. Special reference to stance and gait. Arch Neurol 43(1):34-8

6. Liouta E, Gatzonis S, Kalamatianos T, Kalyvas A, Koutsarnakis C, Liakos F et al (2017) Finger tapping and verbal fluency posttap test improvement in INPH: its value in differential diagnosis and shunt-treatment outcomes prognosis. Acta Neurochir (Wien) 159:2301-2307. https://doi.org/10.1007/s00701-017-3301-2

7. Tsakanikas D, Katzen H, Ravdin LD, Relkin NR (2009) Upper extremity motor measures of Tap Test response in Normal Pressure Hydrocephalus. Clin Neurol Neurosurg 111:752-757. https:// doi.org/10.1016/j.clineuro.2009.07.017

8. Blomsterwall E, Bilting M, Stephensen H, Wikkelsö C (1995) Gait abnormality is not the only motor disturbance in normal pressure hydrocephalus. Scand J Rehabil Med 27:205-209

9. Mandir AS, Hilfiker J, Thomas G, Minahan RE, Crawford TO, Williams MA et al (2007) Extrapyramidal signs in normal pressure hydrocephalus: an objective assessment. Cerebrospinal Fluid Res 4:7. https://doi.org/10.1186/1743-8454-4-7

10. Kang K, Jeon J-S, Kim T, Choi D, Ko P-W, Hwang SK et al (2016) Asymmetric and Upper Body Parkinsonism in Patients with Idiopathic Normal-Pressure Hydrocephalus. J Clin Neurol 12:452. https://doi.org/10.3988/jcn.2016.12.4.452

11. Hellström P, Klinge P, Tans J, Wikkels $\varnothing$ C (2012) The neuropsychology of iNPH: findings and evaluation of tests in the European multicentre study. Clin Neurol Neurosurg 114:130-134. https:// doi.org/10.1016/j.clineuro.2011.09.014

12. Junkkari A, Luikku AJ, Danner N, Jyrkkänen HK, Rauramaa T, Korhonen VE et al (2019) The Kuopio idiopathic normal pressure hydrocephalus protocol: initial outcome of 175 patients. Fluids Barriers CNS 16:21. https://doi.org/10.1186/s12987-019-0142-9

13. Hellström P, Klinge P, Tans J, Wikkelsø C (2012) A new scale for assessment of severity and outcome in iNPH. Acta Neurol Scand 126:229-237. https://doi.org/10.1111/j.1600-0404.2012.01677.x

14. Mathiowetz V, Volland G, Kashman N, Weber K (1985) Adult norms for the Box and Block Test of manual dexterity. Am J Occup Ther 39:386-391

15. Mahoney FI, Barthel DW (1965) Functional evaluation: The Barthel index. Md State Med J 14:61-65

16. Welsh KA, Butters N, Mohs RC, Beekly D, Edland S, Fillenbaum G et al (1994) The Consortium to Establish a Registry for Alzheimer's Disease (CERAD). Part V. A normative study of the neuropsychological battery. Neurology 44(4):609-609. https://doi. org/10.1212/wnl.44.4.609

17. Sotaniemi M, Pulliainen V, Hokkanen L, Pirttilä T, Hallikainen I, Soininen H et al (2012) CERAD-neuropsychological battery in 
screening mild Alzheimer's disease. Acta Neurol Scand 125:1623. https://doi.org/10.1111/j.1600-0404.2010.01459.x

18. Wikkelsø C, Andersson H, Blomstrand C, Lindqvist G (1982) The clinical effect of lumbar puncture in normal pressure hydrocephalus. J Neurol Neurosurg Psychiatry 45:64-69

19. Marmarou A, Bergsneider M, Klinge P, Relkin N, Black PM (2005) The value of supplemental prognostic tests for the preoperative assessment of idiopathic normal-pressure hydrocephalus. Neurosurgery 57(3 Suppl):S17-28

20. Seppälä TT, Nerg O, Koivisto AM, Rummukainen J, Puli L, Zetterberg $\mathrm{H}$ et al (2012) CSF biomarkers for Alzheimer disease correlate with cortical brain biopsy findings. Neurology 78:15681575. https://doi.org/10.1212/WNL.0b013e3182563bd0

21. Mori E, Ishikawa M, Kato T, Kazui H, Miyake H, Miyajima M et al (2012) Guidelines for management of idiopathic normal pressure hydrocephalus: second edition. Neurol Med Chir (Tokyo) 52(11):775-809

22. Klinge P, Marmarou A, Bergsneider M, Relkin N, Black PM (2005) Outcome of Shunting in Idiopathic Normal-pressure Hydrocephalus and the Value of Outcome Assessment in Shunted Patients. Neurosurgery 57(3 Suppl):S40-52. https://doi.org/10. 1227/01.NEU.0000168187.01077.2F

23. Luikku AJ, Hall A, Nerg O, Koivisto AM, Hiltunen M, Helisalmi $S$ et al (2016) Multimodal analysis to predict shunt surgery outcome of 284 patients with suspected idiopathic normal pressure hydrocephalus. Acta Neurochir (Wien) 158:2311-2319. https:// doi.org/10.1007/s00701-016-2980-4

24. Hellström P, Edsbagge M, Blomsterwall E, Archer T, Tisell M, Tullberg M et al (2008) Neuropsychological Effects of Shunt Treatment in Idiopathic Normal Pressure Hydrocephalus. Neurosurgery 63:527-536. https://doi.org/10.1227/01.NEU.00003 25258.16934.BB

25. Yancosek KE, Howell D (2009) A narrative review of dexterity assessments. J Hand Ther 22(3):258-69. https://doi.org/10.1016/j. jht.2008.11.004

26. Laidet M, Herrmann FR, Momjian S, Assal F, Allali G (2015) Improvement in executive subfunctions following cerebrospinal fluid tap test identifies idiopathic normal pressure hydrocephalus from its mimics. Eur J Neurol 22:1533-1539. https://doi.org/10. 1111/ene.12779
27. Gallagher RM, Marquez J, Osmotherly P (2018) Cognitive and upper limb symptom changes from a tap test in Idiopathic Normal Pressure Hydrocephalus. Clin Neurol Neurosurg 174:92-96. https://doi.org/10.1016/j.clineuro.2018.09.015

28. Tsakanikas D, Relkin N (2007) Normal Pressure Hydrocephalus. Semin Neurol 27:058-065. https://doi.org/10.1055/s-2006-956756

29. Kiefer M, Unterberg A (2012) The Differential Diagnosis and Treatment of Normal-Pressure Hydrocephalus. Dtsch Aerztebl Int 109(1-2):15-25. https://doi.org/10.3238/arztebl.2012.0015

30. Sirkka J, Säisänen L, Julkunen P, Könönen M, Kallioniemi E, Leinonen $\mathrm{V}$ et al (2020) Corticospinal excitability in idiopathic normal pressure hydrocephalus: a transcranial magnetic stimulation study. Fluids Barriers CNS 17:6. https://doi.org/10.1186/ s12987-020-0167-0

31. Williams MA, Nagel SJ, Luciano MG, Relkin N, Zwimpfer TJ, Katzen $\mathrm{H}$ et al (2019) The clinical spectrum of hydrocephalus in adults: report of the first 517 patients of the Adult Hydrocephalus Clinical Research Network registry. J Neurosurg 132(6):17731784. https://doi.org/10.3171/2019.2.JNS183538

32. Petersen J, Hellström P, Wikkels $\varnothing$ C, Lundgren-Nilsson Å (2014) Improvement in social function and health-related quality of life after shunt surgery for idiopathic normal-pressure hydrocephalus. J Neurosurg 121:776-784. https://doi.org/10.3171/2014.6.JNS13 2003

33. Junkkari A, Häyrinen A, Rauramaa T, Sintonen H, Nerg O, Koivisto AM et al (2017) Health-related quality-of-life outcome in patients with idiopathic normal-pressure hydrocephalus - a 1-year follow-up study. Eur J Neurol 24:58-66. https://doi.org/10.1111/ ene. 13130

34. Kahlon B, Sjunnesson J, Rehncrona S (2007) LONG-TERM OUTCOME IN PATIENTS WITH SUSPECTED NORMAL PRESSURE HYDROCEPHALUS. Neurosurgery 60:327-332. https:// doi.org/10.1227/01.NEU.0000249273.41569.6E

35. Mlinac ME, Feng MC (2016) Assessment of Activities of Daily Living, Self-Care, and Independence. Arch Clin Neuropsychol 31:506-516. https://doi.org/10.1093/arclin/acw049

Publisher's note Springer Nature remains neutral with regard to jurisdictional claims in published maps and institutional affiliations. 